\section{JTI}

JOURNAL OF

TRAUMA AND INJURY

Received: August 7, 2020

Revised: September 7, 2020

Accepted: September 15, 2020

\section{Correspondence to}

Ye Rim Chang, M.D., Ph.D.

Department of Trauma Surgery, Trauma Center, Dankook University Hospital, 201

Manghyang-ro, Dongnam-gu, Cheonan

31116 , Korea

Tel: +82-41-550-0038

Fax: +82-41-550-0039

E-mail:yrchang@dkuh.co.kr

\title{
Educational Simulation Videos for Performing Resuscitative Endovascu- lar Balloon Occlusion of the Aorta
}

\author{
Sung Wook Chang, M.D., M.S. ${ }^{1,3}$, Dong Hun Kim, M.D., M.S., ${ }^{2,3}$ \\ Ye Rim Chang, M.D., Ph.D. ${ }^{2,3}$ \\ ${ }^{1}$ Department of Thoracic and Cardiovascular Surgery, Trauma Center, Dankook University \\ Hospital, Cheonan, Korea \\ ${ }^{2}$ Department of Trauma Surgery, Trauma Center, Dankook University Hospital, Cheonan, \\ Korea \\ ${ }^{3}$ Korean Association for Research, Procedure and Education on Trauma (KARPET), Seoul, \\ Korea
}

Resuscitative endovascular balloon occlusion of the aorta (REBOA) has been accepted as an adjunct procedure for non-compressible torso hemorrhage in patients with hemorrhagic shock. With appropriate indications, REBOA should be performed for resuscitation regardless of the physician's specialty. Despite its effectiveness in traumatized patients with hemorrhagic shock, performing REBOA has been challenging due to physicians' lack of experience. Even though training in endovascular skills is mandatory, many physicians cannot undergo sufficient training because of the limited number of endovascular simulation programs. Herein, we share simulation video clips, including those of a vascular circuit model for simulation; sheath preparation; long guidewire and balloon catheter preparation; ultrasound-guided arterial access; sheath insertion or upsizing; and balloon positioning, inflation, and migration. The aim of this study was to provide educational video clips to improve physicians' endovascular skills for REBOA.

Keywords: Trauma; Resuscitation; Aorta; Balloon occlusion; Instructional video

\section{INTRODUCTION}

Resuscitative endovascular balloon occlusion of the aorta (REBOA) has been developed to manage non-compressible torso hemorrhage in trauma patients with hemorrhagic shock [1-3]. Currently, the use of REBOA is widely accepted, as several studies 
have reported that REBOA could be an adjunct procedure for resuscitation in patients with neurogenic shock and impending non-traumatic cardiac arrest to support the maintenance of central aortic pressure, as well as brain and heart perfusion [4-7]. Despite its effectiveness, the application of REBOA remains limited owing to physicians' lack of experience [2,8-11]. To overcome this limitation, several educational courses for REBOA have been developed, such as the endovascular skills for trauma and resuscitative surgery (ESTARS) course and the endovascular resuscitation, bleeding, and trauma management (EVTM) hands-on workshop. In South Korea, the endovascular training for REBOA (ET-REBOA) course has been developed and conducted since 2017. Unfortunately, educational programs are difficult to access due to limited resources, which makes it challenging for physicians to acquire the skills for REBOA. The aim of this article was to share simulation video clips to aid preparedness and to impart endovascular skills for REBOA. The Blue Phantom $^{\text {TM }}$ (Femoral Vascular Access Lower Torso Ultrasound Model, CAE Healthcare, Saratoga, FL, USA) and $\mathrm{EVE}^{\mathrm{TM}}$ vascular circuit models (Endo Vascular Evaluator, BR Biomedicals Pvt. Ltd., New Delhi, India; Supplementary Video 1) were used for vascular access and balloon manipulation, respectively. The 5-/7-Fr Radiofocus ${ }^{\circledR}$ Introducer II (Terumo, Tokyo, Japan) and 7-Fr RESCUE balloon $^{\mathrm{TM}}$ catheter (Tokai Medical Products, Aichi, Japan) were used in the video simulation.

\section{MAIN BODY}

\section{Sheath preparation}

The sheath set consists of a puncture needle, a miniwire, a dilator, and a sheath (Supplementary Video 2). Before inserting the vascular sheath, the dilator and sheath should be primed with heparinized or normal saline $[9,12]$. If priming is not performed, resistance between the dilator and sheath may cause them to break. A damaged dilator tip and sheath can lead to a vascular injury during the introduction of the sheath, and secondary dysfunction might occur while passing the balloon catheter. After priming, the dilator should be fully inserted in the sheath and fitted until it clicks to prevent slipping of the sheath.

\section{Long guidewire and balloon catheter preparation}

During preparation of the sheath, a long guidewire and a 7-Fr RESCUE balloon ${ }^{\mathrm{TM}}$ catheter should be sufficiently primed by flushing with saline to activate the hydrophilic coating along the guidewire and balloon catheter (Supplementary Video 3). A high level of resistance may prevent the insertion of a long guidewire into the central lumen of the balloon catheter if the priming process is not performed, which makes REBOA impossible.

During balloon catheter preparation, a balloon test must not be performed through the plug of the 3-way stopcock. The diameter of the balloon portion of the balloon catheter is enlarged if the balloon test is performed; therefore, passing it through the 7-Fr sheath becomes difficult. Even if the balloon catheter is fully deflated with an accurate maneuver after testing, it cannot be completely deflated to a state similar to that before the test. The balloon may be damaged even if it passes through the 7-Fr sheath successfully. To solve this problem, the sheath must be changed to a new one that is larger than 8 or $9 \mathrm{Fr}$ or a new balloon catheter must be prepared for use with the previous 7-Fr sheath.

\section{Ultrasound-guided arterial access}

As the common femoral artery (CFA) is located superficially, it is easy to access by palpation, and its diameter is relatively large, helping to avoid distal ischemia; thus, it is preferred over the superficial femoral artery (SFA) [13]. However, the Seldinger technique may be difficult to perform in patients with profound shock and obesity as it may lead to vascular access site complications $[13,14]$. To establish safe vascular access, ultrasound-guided arterial access is recommended as the most effective modality $[2,14]$. A prepuncture ultrasonographic examination to identify the bifurcation between the CFA and SFA is a non-time-consuming and valuable procedure $[12,14]$.

For approaching the CFA, ultrasonography can be performed in the transverse and longitudinal views. A transverse view can help visualize the femoral artery and vein and identify the division of the CFA into the SFA and deep femoral artery. However, the location of the needle tip during the procedure may be difficult to track because it is only visible as a dot. If the ultrasound transducer is turned $90^{\circ}$ to obtain a longitudinal view after identifying 
the CFA, it may make arterial puncture easier without injuring the posterior vascular wall (Supplementary Video 4). Regardless of which ultrasonographic approach is chosen for visualization of the CFA, ultrasonography should be readily available at all times and should be used during vascular access.

\section{Sheath insertion or upsizing}

When physicians encounter trauma patients with profound shock, they have to decide whether REBOA is needed. Since the progression of shock can make vascular access difficult, the decision regarding vascular access should be made as quickly as possible [2,15]. If a physician decides to perform REBOA, a 7-Fr or larger sheath should be inserted first (Supplementary Video 5). When the decision regarding REBOA has not been made after the initial resuscitation, a 5-Fr sheath can be inserted in advance. The 5-Fr sheath can be used for diagnostic intervention or for upsizing to complete the REBOA procedure. The sheath must be changed to one of a suitable size because a 7-Fr or larger sheath is required to introduce the 7-Fr RESCUE balloon catheter (Supplementary Video 6). If a micropuncture set (e.g., Micropuncture ${ }^{\circledast}$ Access Set, Cook Medical, Bloomington, IN, USA; outer catheter, 4-Fr; guidewire diameter, 0.018 in; needle gauge, 21 ) is available, it can be used for vascular access in patients with profound shock.

\section{Balloon positioning and inflation}

Before positioning the balloon catheter at the target level,

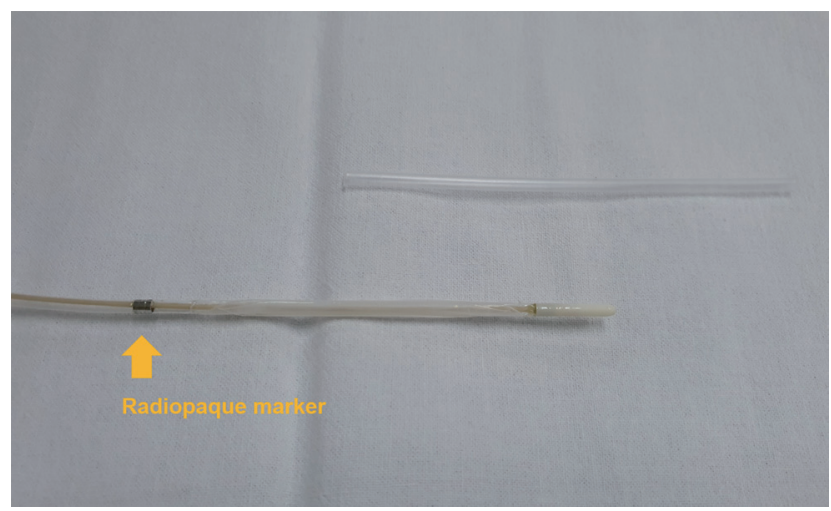

Fig. 1. A radiopaque steel marker at the lower end of the balloon of the 7-Fr RESCUE balloon ${ }^{\mathrm{TM}}$ catheter (Tokai Medical Products, Aichi, Japan). the position of the long guidewire must be visualized using fluoroscopy or radiography. If the guidewire is properly placed in the aorta (at least in the thoracic descending aorta), a balloon catheter can be introduced along the guidewire. To minimize the risk of ischemic complications, accurate balloon positioning is important: aortic Zone I for patients with intra-abdominal hemorrhage and aortic Zone III for patients with unstable pelvic fractures [2]. If radiological equipment is not available, external landmarks may be used as substitutes in urgent situations. The radiopaque landmark of the balloon catheter should be located above the xyphoid process for Zone I REBOA and near the umbilicus for Zone III REBOA (Fig. 1). However, the estimated length of the balloon catheter is not always accurate for aortic occlusions, and the inflated balloon catheter may migrate in a caudal direction; therefore, the position of the balloon catheter should be checked before and after inflation (Supplementary Video 7). In particular, if a guidewire is not changed to a stiff stylet before inflation, the inflated balloon might migrate or bend due to elevated blood pressure (Supplementary Video 8). Therefore, this process is important for accurately placing the balloon catheter at the target level. A 7-Fr RESCUE balloon catheter can be inflated to 40 $\mathrm{mm}$ in diameter with $40 \mathrm{~mL}$; however, the balloon should be inflated according to the patient's status and targeted blood pressure since the aorta is usually collapsed in patients with hemorrhagic shock.

\section{CONCLUSION}

Management strategies for patients with hemodynamic instability have changed in the last few decades. Among these strategies, the development of REBOA represents a paradigm shift. Even though REBOA is not a definitive therapy, but a bridging therapy to save lives, the role of REBOA should be sufficiently emphasized to prevent trauma-related deaths. Therefore, on the basis of accurate indications, REBOA should be performed regardless of the patient's location and the physician's specialty. We believe that this article, with its simulation videos, will aid in preparedness for performing REBOA. 


\section{SUPPLEMENTARY MATERIALS}

The online-only data supplement is available with this article at https://doi.org/10.20408/jti.2020.0035.

\section{REFERENCES}

1. Stannard A, Eliason JL, Rasmussen TE. Resuscitative endovascular balloon occlusion of the aorta (REBOA) as an adjunct for hemorrhagic shock. J Trauma 2011;71:1869-72.

2. Kim DH, Chang SW, Matsumoto J. The utilization of resuscitative endovascular balloon occlusion of the aorta: preparation, technique, and the implementation of a novel approach to stabilizing hemorrhage. J Thorac Dis 2018;10:5550-9.

3. Bulger EM, Perina DG, Qasim Z, Beldowicz B, Brenner M, Guyette F, et al. Clinical use of resuscitative endovascular balloon occlusion of the aorta (REBOA) in civilian trauma systems in the USA, 2019: a joint statement from the American College of Surgeons Committee on Trauma, the American College of Emergency Physicians, the National Association of Emergency Medical Services Physicians and the National Association of Emergency Medical Technicians. Trauma Surg Acute Care Open 2019;4:e000376.

4. Daley J, Morrison JJ, Sather J, Hile L. The role of resuscitative endovascular balloon occlusion of the aorta (REBOA) as an adjunct to ACLS in non-traumatic cardiac arrest. Am J Emerg Med 2017;35:731-6.

5. Gray S, Dieudonne B. Resuscitative endovascular balloon occlusion of the aorta as an adjunct in a patient with neurogenic shock. Cureus 2018;10:e3375.

6. Chang SW, Chang YR, Seon YJ, Park JW, Kim JS. Intraoperative use of resuscitative endovascular balloon occlusion of the aorta for hemorrhage control in woman with placenta percreta involving the bladder. Perinatology 2019;30:175-8.
7. Andres J, Scott J, Giannoudis PV. Resuscitative endovascular balloon occlusion of the aorta (REBOA): what have we learned? Injury 2016;47:2603-5.

8. Villamaria CY, Eliason JL, Napolitano LM, Stansfield RB, Spencer JR, Rasmussen TE. Endovascular skills for trauma and resuscitative surgery (ESTARS) course: curriculum development, content validation, and program assessment. J Trauma Acute Care Surg 2014;76:929-935; discussion 935-6.

9. Kim DH, Chang SW. Resuscitative endovascular balloon occlusion of the aorta: focusing on the procedure. Trauma Image Proced 2017;2:92-3.

10. Vernamonti JP, Holcomb J, Mick NW, Falank C, Ontengco JB, Rappold J, et al. 'Step Up' approach to the application of REBOA technology in a rural trauma system. Trauma Surg Acute Care Open 2019;4:e000335.

11. Zakaluzny SA, Beldowicz BC, Salcedo ES, DuBose JJ, Moore LJ, Brenner M. Guidelines for a system-wide multidisciplinary approach to institutional resuscitative endovascular balloon occlusion of the aorta implementation. J Trauma Acute Care Surg 2019;86:337-43.

12. Kandarpa K, Machan L, Durham J. Handbook of interventional radiologic procedures. 5th ed. Philadelphia: Wolters Kluwer; 2016.

13. Ahn HY, Lee HJ, Lee HJ, Yang JH, Yi JS, Lee IW. Assessment of the optimal site of femoral artery puncture and angiographic anatomical study of the common femoral artery. J Korean Neurosurg Soc 2014;56:91-7.

14. Son SY, Cho KC, Cho P, Lee JH, Myoung SU, Choi JH. Prepuncture ultrasound examination facilitates safe and accurate common femoral artery access for transfemoral cerebral angiography. J Cerebrovasc Endovasc Neurosurg 2017;19:276-83.

15. Matsumoto J, Lohman BD, Morimoto K, Ichinose Y, Hattori T, Taira Y. Damage control interventional radiology (DCIR) in prompt and rapid endovascular strategies in trauma occasions (PRESTO): a new paradigm. Diagn Interv Imaging 2015;96:687-91. 
Sung Wook Chang, et al. Simulation Videos for Performing REBOA

Supplementary Video 1. Preview of the EVE ${ }^{\mathrm{TM}}$ (Endo Vascular Evaluator, BR Biomedicals Pvt. Ltd., New Delhi, India) model for the simulation of REBOA. 
JTI

Journal of Trauma and Injury Volume 33, Number 3, September 2020

Supplementary Video 2. Preparation of a 7-Fr sheath introducer. 
Sung Wook Chang, et al. Simulation Videos for Performing REBOA

Supplementary Video 3. Preparation of a 7-Fr balloon catheter and its long guidewire. 
JTI

Journal of Trauma and Injury Volume 33, Number 3, September 2020

Supplementary Video 4. Methodology of ultrasound-guided femoral arterial access. 
Sung Wook Chang, et al. Simulation Videos for Performing REBOA

Supplementary Video 5. Insertion of a 7-Fr sheath introducer in Blue Phantom ${ }^{\mathrm{TM}}$ (Femoral Vascular Access Lower Torso Ultrasound Model, CAE Healthcare, Saratoga, FL, USA). 
JTI

Journal of Trauma and Injury Volume 33, Number 3, September 2020

Supplementary Video 6. Upsizing of the sheath introducer from 5-Fr to 7-Fr. 
Sung Wook Chang, et al. Simulation Videos for Performing REBOA

Supplementary Video 7. Practical simulation of REBOA using the EVE ${ }^{\mathrm{TM}}$ (Endo Vascular Evaluator, BR Biomedicals Pvt. Ltd., New Delhi, India) model. 
JTI

Journal of Trauma and Injury Volume 33, Number 3, September 2020

Supplementary Video 8. Migration of a balloon catheter without an intrinsically stiff stylet. 